\title{
Role of Nutrition in Occupational Health and Safety in the context of COVID-19
}

\author{
Sunuwar DB ${ }^{1}$ \\ ${ }^{1}$ Chairperson, Holistic Health Private Limited, Chabahil, Kathmandu, Nepal
}

$\mathrm{O}$ ccupational health and safety (OHS) is a multidisciplinary field concerned with enabling an individual to undertake their occupation, in a way that causes the least harm to their health.

OHS focuses on three different objectives: maintenance and promotion of workers' health and working capacity; improvement of working environment and work; and development of work organizations and working cultures in a direction which supports health and safety at work.

At the workplace, workers are exposed to different hazards that can adversely affect their health and well-being. Long hours, changing shifts, physically demanding tasks, violence, insufficient nutrition and exposures to stress and chemicals are examples of hazards that put these workers at risk for illness and injury.

The most recently discovered SARS Coronavirus2 causes "COVID-19" which was unknown before the outbreak began in Wuhan, China, in December 2019. It has now turned to a pandemic state affecting many countries globally.

People can contract COVID-19 from other persons who have the virus. The disease spreads primarily from person to person through respiratory droplets, which are expelled from the nose and mouth when a person with COVID-19 coughs, sneezes or speaks. These droplets being relatively heavy, do not travel far and quickly sink to the ground. In the context of COVID-19,

DOI: https://doi.org/10.3126/ijosh.v10i1.29875

\section{Corresponding Author}

Dambar Bahadur Sunuwar

Chairperson,

Holistic Health Private Limited, Chabahil

Kathmandu, Nepal

Tel: 9851063742

Email: drsunuwardb@gmail.com it has added extra health risks to the workers working in an overcrowded environment.

Nutrition is the science that studies the role of micro and macronutrients in food concerning maintenance, growth and reproduction of an organism. It also plays an important role in health and disease of the workers. The diet of the workers is largely determined by the availability and palatability of foods. For healthy workers, a proper diet includes preparation of food and storage methods that preserve nutrients from oxidation, heat or leaching, and that reduces the risk of foodborne illnesses.

In the workers, an unbalanced diet can cause a wide range of deficiency-related diseases such as iron deficiency anemia, blindness, scurvy, hypothyroidism, obesity, cardiovascular disease, and diabetes etc. Undernutrition can lead to wasting in acute cases, and the stunting in chronic cases of malnutrition.

Without proper nutrition, the workers cannot perform well at work. Whether the workers are from the health sector, or construction sector, or any other sector, nutritious food is the fundamental requirement for productive work. During the COVID-19 pandemic, nutrition has proved to be an important factor to fight against the infection. Compared to the normal work routine, workers are being held at their workplaces for increased work hours. It means, there is some extra requirement for nutrition. They require nutritious food to properly function their body, to increase their working capacity. Studies have revealed that people with weak immune status are more susceptible to COVID-19 and its complications. It is a well-known fact that proper nutrition is largely associated with good immunity status. Healthcare workers recommend the use of antioxidants and anti-inflammatory compounds

\section{(c) (1) (8)}

This journal is licensed under a Creative Commons AttributionNon Commercial 4.0 International License. 
rich foods to improve the immunity of the general population. It is wise to select and include food materials containing these compounds in daily diet to increase one's immunity power.

It has also been proved that COVID-19 harms people with chronic diseases such as cardiovascular disease, diabetes, renal diseases etc. Those diseases are to some extent related to the nutritional status.

Different functional foods that boost the immunity power are available in the market. Supplementary foods such as Spirulina, Ganoderma, Cordyceps, Noni, Curcumin, etc. are recommended to improve the immunity power, though such food supplements have not been tested yet among the COVID-19 patients.

Proper nutrition is the basis of a healthy lifestyle and an efficient work output. It needs to be considered that diet should be balanced to fight the risks of COVID-19 since only the preventive measures need to be applied against it in this present context. 